part in other specialties and in general practice. In geriatrics, for example, nurses might run major parts of the hospital service, calling in a consultant or GP only for specialist medical advice. The increasing interest in this expanded clinical role of a nurse may exacerbate or help the persistent shortage of nurses, but pilot schemes should rapidly resolve this important question.

General practitioners also share the hospital service load and many more are entering hospitals to look after patients who are normally in their care as outpatients and this is reflected in the recently created hospital practitioner grade. Further expansion of this development would integrate the Health Service more effectively than the recent reorganisation.

\section{Future solutions}

Our policy concerning medical manpower requires urgent action. At present we can muddle through only by starving many consultants of what they would regard to be their proper junior staff establishment, by the dedication of junior doctors working long hours, and by the grace of overseas doctors who are carrying out many of the tasks of a service doctor or long service SHO but cannot be relied on in the future to bail us out of our present inconsistent policies. Any future policy must take into account the unpalatable statistical fact that the country's medical schools can never produce sufficient graduates to meet our present aspirations for junior hospital staffing on the basis of the present organisation of hospital grades and training programmes. Once this has been firmly grasped a rigorous look must follow at the pattern of service needs and the best way of meeting those from our supply of medical graduates and paramedical staff. Medical schools must also look again at the arguments in favour of increasing their output.
In future consultants, nurses, and GPs will have to assume more of a service role to enable the number of junior staff to be trimmed radically into line with available career posts. There is no case for maintaining too many junior doctors unless British doctors are prepared to accept a permanent subconsultant grade, an enforced stay at SHO level, or a career grade hierarchy. The only realistic alternative to these solutions is a rundown of junior staff.

Such a rundown can be helped by transferring many of those posts to consultant and GP establishments. This would reduce the disparity between the numbers of training and permanent posts and also provide both groups with additional staff to help them take on hospital service tasks. In effect, the number of general practitioners could be considerably increased by such a transfer and this would enable the size of lists to fall and thus free time for hospital duties. The increase in consultants would also have the same effect.

Closing uneconomic hospitals will also be necessary and an expansion of the role of nurses into clinical care will have to be fully explored. The combination of extra consultants, more general practitioners, and newly created nurse clinicians should fill the gap produced by trimming back junior hospital posts to a size sufficient to meet training needs and actuarial imperatives.

I am indebted to Mr F S A Doran for his help and encouragement and to my colleagues who have provided helpful advice. This article reflects solely my personal views.

Guy's Hospital, London SE1 9RT

T J H CLARK, MD, FRCP, consultant physician

\title{
The ideal health centre?
}

\section{J C DUNBAR}

Priority given to clinical efficiency in the provision of preventive and primary health care ensures maximum administrative and economic efficiency. This paper illustrates this fact, which is repeatedly overlooked by administrators, by describing the cheapest and most efficient health centre encountered during a 12-year peripatetic international career in general practice.

The health centre was a cross between a conventional general practice and a cottage hospital. It served a population of about 10000 living in an area of $1295 \mathrm{~km}^{2}$. The economy was agricultural and industrial-oil and natural gas production.

\section{Facilities and staff}

There was a family doctor service backed up by a 24-hour casualty service; 12 beds; an $x$-ray department capable of handling intravenous pyelograms and cholecystograms in addition to routine views; a laboratory in which the most expensive equipment consisted of a microscope, incubator, and microbalance and which was capable of handling the investigations listed in table $\mathrm{I}$; a pharmacy which, in addition to stocking the usual proprietary preparations, was better equipped than most to compound old but none the less cheap and effective remedies; a minor operating theatre, which was not equipped with an anaesthetic machine, thus placing an effective limit on the pretensions of the unit; and a modern dental surgery and workshop.

A staff of 26 operated the centre. There were a doctor, dentist, nursing sister/matron, two charge nurses (one of whom had the responsibilities of a tutor), a pharmacist, an $x$-ray/laboratory technician, a lay administrator, four shift nurses, two medical dressers, three medical orderlies, six ward orderlies, and three sanitary orderlies. There was also a recently qualified nurse working as a trainee in the special nursing requirements of the unit.
TABLE I-Available laboratory investigations

\begin{tabular}{l}
\hline Blood \\
Hb, full blood count, and differential \\
ESR \\
Smear for malarial parasites \\
Group and Rh factor \\
Widal \\
Kahn \\
Sugar (random and GTT) \\
Urea \\
Uric acid \\
Some liver function tests \\
Sputum \\
Direct Gram and Ziehl Neelsen stains for AFB \\
Culture and sensitivity \\
Urine \\
Routine analysis and microscopic examination \\
Culture and sensitivity \\
Pregnancy test \\
Stool \\
Occult blood \\
Microscopic for parasites \\
Culture and sensitivity
\end{tabular}

The centre coped with all of the primary medical care of the community and $99 \%$ of the secondary medical care-that is, only $1 \%$ of the presenting illnesses were referred to the general hospital $81 \mathrm{~km}$ away for specialist investigation and treatment. So the unit prevented expensive general hospital facilities from being abused, leaving its staff free to concentrate on serious illnesses.

\section{Casualty department}

The hub of the centre was the casualty department. It was manned by the shift nurses, who also cared for any patients in the wards. They, 
together with the other staff, were helped by the orderlies. The sister and charge nurses provided overall supervision. The charge nurses had basic experience in radiography, the pathological laboratory, pharmacy, and administration and so provided holiday relief in these departments.

All patients reported first to the duty nurse. She was not expected to make an accurate diagnosis but rather to assess the severity of the complaint. A decision could then be made whether or not to call the doctor. Experience usually enabled the nurse to diagnose and treat most cases and the doctor was seldom disturbed after he had gone. Night calls averaged three a year. All the nurses were competent in resuscitation if this was necessary before the doctor arrived.

The doctor performed most of his clinical work in a three-hour surgery every weekday morning after his ward round. This left afternoons free for routine industrial medical examinations, correspondence, public health responsibilities, and study. Being single handed, he was never off duty.

The sister screened all women and preschool children. She ordered simple investigations and prescribed simple remedies. Consequently the doctor saw only three or four women and children a day and was spared the task of treating minor infections, domestic neuroses, etc. Moreover, since mothers of sick children, particularly from poorer "high risk" homes, tend to bring all their offspring, the sister effectively operated a "well-baby clinic."

\section{Role of the community}

The community shared in the responsibility for providing its own care. An ambulance, owned, maintained, and manned by the local garage, was on 24-hour standby. The doctor's car was maintained for his sole use by the local taxi proprietor. Meals for inpatients were provided by the local restaurant. Few visits were required, as relatives and neighbours were encouraged to transport patients to the centre themselves. Patients were not encouraged in delusions of grandeur or to make their ailments a matter of convenience by an appointments system. To minimise waiting different categories of patients were seen at specified times-for example, manual workers were seen first after the ward round.

The efficiency of the unit is shown by the fact that any investigations ordered for patients had usually been completed by late morning so that all the presenting illnesses of the day had started treatment by lunchtime. Patients had no opportunity for hypochondriacal rumination. Moreover, patients could seek advice at any time without fear of unnecessarily disturbing the doctor. The work load and cost of running the centre are analysed in table II.

TABLE II-Analysis of work load and running costs for week beginning 10/2/1974

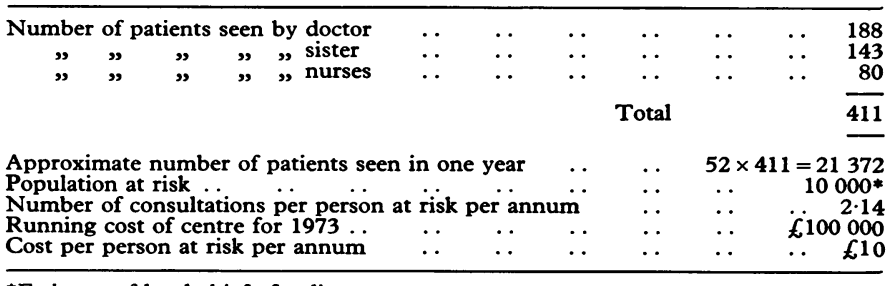

*Estimate of local chief of police.

\section{Comment}

The unit demonstrated an anomaly overlooked by administrators the world over. The salary of the doctor and the dentist was $£ 10000$ and that of the orderlies about $£ 2000$. The doctor was on call 168 hours a week and had a work responsibility four times greater than everyone else. He was therefore paid a quarter of the salary of the dentist and little more than that of an orderly. The anomaly would be rectified by employing two doctors at $£ 20000$ each.

Some people feel that a health centre administered along these lines is wide open to the depredations of litigants. Nevertheless, it is a fact of human nature that even the most ardent litigants will forgive accidental errors so long as they feel that they have been treated with kindness, care, and thoughtful consideration.

London SW5 0ET

I J C DUNBAR, MB, CHB, general practitioner

\section{Scottish GMS Committee}

The Scottish GMS Committee met on 4 November with Dr Joan $\mathrm{K}$ Sutherland in the chair.

\section{Vocational training}

The committee rejected a suggestion from the Scottish Council for Postgraduate Medical Education that a panel of assessors be appointed to advise on the quality of future entrants as principals in general practice. It also rejected a proposal that six months' GP experience should be mandatory for acceptance as a principal in general practice.

The definition of "loss of remunerative time" continued to cause the committee concern and there are to be more discussions with the Scottish Home and Health Department in an attempt to obtain payment for those GPs who spend a lot of time on NHS committees.

\section{Removal expenses and mileage payments}

The Scottish Home and Health Department has refused to meet the removal expenses of a practitioner who wishes to leave an "inducement" practice, though similar expenses are reimbursed on the "outward" journey. This disincentive will be examined further by the rural subcommittee. The subcommittee will also look at the suggestion that the Review Body might make money available to meet the cost of paying a mileage allowance for attendance on temporary residents.

\section{Nurses}

The committee expressed concern that central nursing offices held detailed nursing records. Many records contain personal details and this possible breach of confidentiality will be discussed in the National Medical Consultative Committee and in the Scottish Council's Liaison Committee with the Royal College of Nursing.

The committee was told that an area health board had refused permission for its district nurses to do influenza immunisations in patients' homes. So general practitioners may have to make extra visits even though the nurse may be in attendance for other nursing procedures. The committee pointed out that this would increase GPs' work load and asked for the matter to be considered further. 